\title{
Serum Cystatin C, Markers of Chronic Kidney Disease, and Retinopathy in Persons with Diabetes
}

\author{
Chee Wai Wong, ${ }^{1}$ Boon Wee Teo, ${ }^{2}$ Ecosse Lamoureux, ${ }^{1,3,4}$ \\ Mohammad Kamran Ikram, ${ }^{1,4}$ Jie Jin Wang, ${ }^{5}$ E. Shyong Tai, ${ }^{2}$ Sunil Sethi, ${ }^{6}$ \\ Tien Yin Wong, ${ }^{1,3,4}$ and Charumathi Sabanayagam ${ }^{1,3,4}$

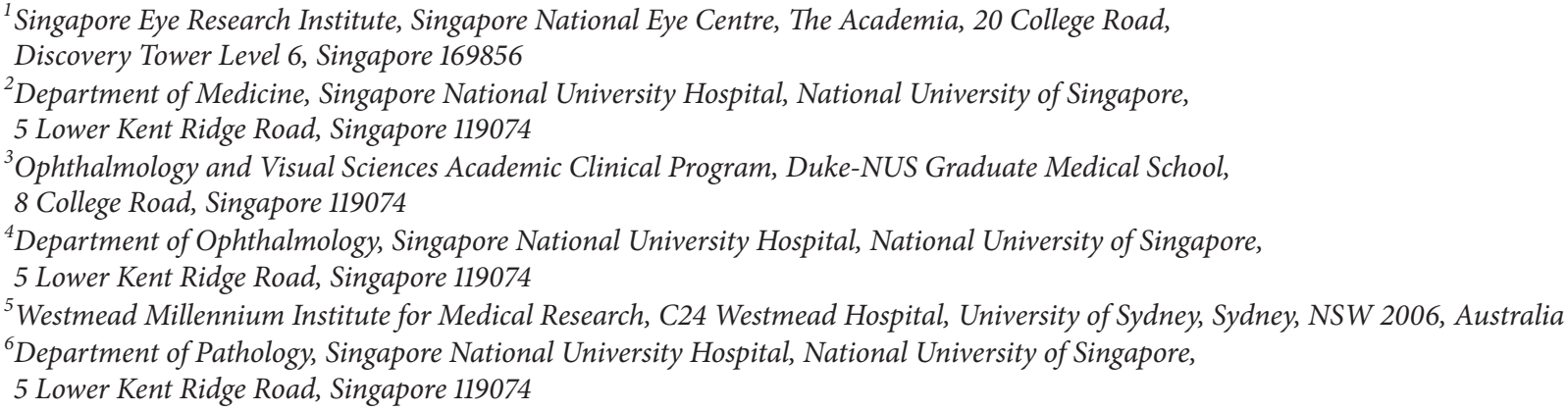

Correspondence should be addressed to Charumathi Sabanayagam; charumathi.sabanayagam@seri.com.sg

Received 4 November 2014; Accepted 13 January 2015

Academic Editor: Francesco Chiarelli

Copyright (c) 2015 Chee Wai Wong et al. This is an open access article distributed under the Creative Commons Attribution License, which permits unrestricted use, distribution, and reproduction in any medium, provided the original work is properly cited.

\begin{abstract}
Purpose. We examined the association of CKD defined by serum creatinine, serum cystatin C, and albuminuria with moderate diabetic retinopathy (DR). Methods. We examined 1,119 Indian adults with diabetes, aged 40-80 years, who participated in the Singapore Indian Eye Study (2007-2009), a population-based cross-sectional study. The associations of CKD defined by each of the three markers alone and in combination with moderate DR were examined using logistic regression models adjusted for potential confounding factors including duration of diabetes, smoking, body mass index, systolic blood pressure, and HbAlc. Results. The prevalence of moderate DR was significantly higher among those with CKD defined by triple markers (41.1\%) compared to CKD defined separately by creatinine (26.6\%), cystatin C (20.9\%), and albuminuria (23.4\%). People with CKD defined by triple markers had a fourteenfold higher odds of moderate DR $(\mathrm{OR}(95 \% \mathrm{CI})=13.63(6.08-30.54))$ compared to those without CKD by any marker. Nearly half (48.7\%) of participants with cystatin C $\geq 1.12 \mathrm{mg} / \mathrm{L}$ have moderate DR. Conclusions. CKD defined by a triple marker panel was strongly associated with moderate DR in this Asian population with diabetes.
\end{abstract}

\section{Introduction}

Diabetic retinopathy (DR), a microvascular complication of diabetes mellitus (DM), is a global public health problem affecting an approximate of 93 million people worldwide, 28 million of whom suffer from vision threatening DR [1]. In Asian countries, the prevalence of DR among those with diabetes ranges from 15.8 to $43.1 \%$ [2-4]. With the rapidly rising prevalence of DM in Asian countries like China and India [5] and an ageing population, the socioeconomic burden of DR is likely to increase exponentially in the near future. Chronic kidney disease (CKD), another major complication of diabetes, is also on the rise worldwide. In diabetic patients, diabetic nephropathy (DN) defined by the presence of albuminuria has been shown to be associated with DR in several studies [6-10]. The concordance between DN and DR could be explained by the sharing of common risk factors including glycemic control, duration of diabetes, blood pressure control, and common pathogenic pathways between the two microvascular complications [11]. 


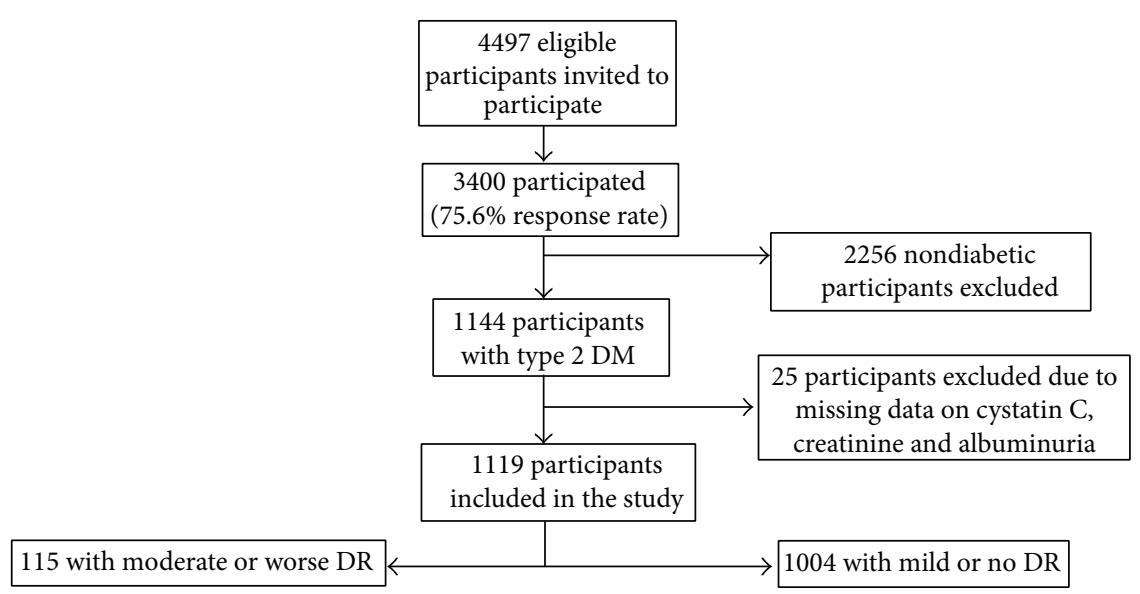

FIGURE 1: Flow diagram of study enrolment. All participants of Indian ethnicity were eligible unless he/she had moved from the residing address, had not lived at the official address in the past six months, or was terminally ill (e.g., cancer) or deceased.

In addition, genetic risk factors have also been shown to contribute to the concordance between DN and DR $[12,13]$. Genetic variants such as CPVL/CHN, rs 39059, associated with $\mathrm{DN}[14,15]$ have also been shown to be associated with DR $[16,17]$. The cannabinoid type 1 receptor gene polymorphism (G1359A in CNR1 gene), shown to be significantly associated with DR and DN in type 2 diabetics [18], suggests another pathogenic link between nephropathy and retinopathy. However, this concordance between albuminuria and DR has been shown to be stronger in type 1 diabetic patients. In type 2 diabetes, although similar association has been documented $[6,9,10,19]$, the discordance between the two has been shown to be frequent due to the coexistence of nondiabetic kidney disease. Recent studies have shown that, in diabetic patients, CKD is less likely to be associated with DR in the absence of albuminuria $[6,9,10]$.

In current clinical practice, CKD is defined by glomerular filtration rate estimated from serum creatinine. Recently, serum cystatin $\mathrm{C}$, an alternative marker of kidney function, either alone or in combination with serum creatinine and/or albuminuria has been shown to be a better predictor of adverse outcomes than serum creatinine alone [20-25]. Serum cystatin $C$ is a cysteine protease inhibitor found in virtually all human tissues and body fluids, which, in contrast to creatinine, is less affected by age, race, or muscle mass $[24,26]$. A clinic based study from China has shown that elevated levels of cystatin C are associated with severity of DR and are an independent risk factor for DR along with diabetes duration and HbAlc levels [27]. A double marker approach combining serum creatinine and albuminuria has been shown to be strongly associated with DR compared to creatinine alone in recent studies $[6,9,10]$. However, the association of CKD defined by a triple marker panel including cystatin $\mathrm{C}$ in addition to creatinine and albuminuria with DR has not been evaluated before. The association with DR could be stronger either by better classification of patients into CKD categories or by virtue of being markers for different pathologic processes in DR, independent of GFR mechanisms. In this context, we examined the association of CKD defined by triple markers with diabetes-specific moderate retinopathy in a population-based sample of Asian adults with diabetes. The aim of this study was to determine whether CKD defined by all triple markers (creatinine, albuminuria, and cystatin C) was more strongly associated with moderate DR compared to each marker in isolation or in dual combination.

\section{Materials and Methods}

2.1. Study Population and Design. The current study is based on data derived from the Singapore Indian Eye Study (SINDI), a population-based cross-sectional study of 3,400 Indian adults aged 40-80 years living in Singapore between 2007 and 2009. Details of the study design, sampling plan, and methodology have been reported elsewhere [28]. In brief, 6,350 adults were selected by an age-stratified random sampling method from the computer generated random list of 11,616 Indian names provided by the Ministry of Home Affairs. Of the 4,497 eligible participants, 3,400 participated in the study (75.6\% response rate). Of these, 1,144 participants had diabetes mellitus defined as random glucose of $11.1 \mathrm{mmol} / \mathrm{L}$ or more, use of diabetic medication, or a physician diagnosis of diabetes mellitus or HbAlc > $6.5 \%(47.5 \mathrm{mmol} / \mathrm{mol})$. After excluding those with missing information on cystatin C, creatinine, albuminuria, and other variables included in the multivariable model $(n=25), 1,119$ participants with diabetes mellitus were included in the current study (Figure 1). The study was conducted in accordance with the Declaration of Helsinki and approval for the study was granted by the National Healthcare Group (2012/00291) and Singhealth (2012/377/A) Institutional Review Boards. Written informed consent was obtained from all participants before enrolment.

2.2. Study Procedures. Standardized systemic and ocular examinations, interviewer-administered questionnaires, and standard blood investigations were conducted for all participants. A detailed interviewer-administered questionnaire was used to collect relevant demographic data and medical history from all participants. Alcohol drinkers were defined by the consumption of alcohol at least once a week. 
Blood pressure was measured with a digital automatic blood pressure monitor (Dinamap model Pro Series DP110X-RW, 100V2; GE Medical Systems Information Technologies, Inc., Milwaukee, WI) after the participants were seated for at least 5 minutes. Venous blood samples were collected for biochemistry tests, including serum lipids (total cholesterol, highdensity lipoprotein cholesterol, and low-density lipoprotein cholesterol), glycosylated hemoglobin Alc (HbAlc), creatinine, and random glucose. The average of the 2 systolic and diastolic blood pressure measurements was used as the systolic and diastolic blood pressure value. Hypertension was defined as systolic blood pressure of $140 \mathrm{mmHg}$ or more or diastolic blood pressure of $90 \mathrm{mmHg}$ or more or self-reported physician-diagnosed hypertension. Diabetes mellitus was defined as random glucose of $11.1 \mathrm{mmol} / \mathrm{L}$ or more, use of diabetic medication, or a physician diagnosis of diabetes mellitus $[29,30]$. We did not differentiate between type 1 or type 2 diabetes. However, since $95 \%$ of our participants reported having their diabetes diagnosed after the age of 35 years, we assume that the majority had type 2 diabetes.

2.3. Assessment of $D R$. Fundus photography was performed using a digital nonmydriatic retinal camera (Canon CRDGi with a 20Diopter SLR backing, Canon, Japan) using early treatment for diabetic retinopathy study (ETDRS) standard field 1 (centered on the optic disc) and ETDRS standard field 2 (centered on the fovea). DR was evaluated following a standard protocol based on retinal photographs which were graded according to a modified scale from the Airlie House Classification System by trained graders [31]. Moderate DR was defined to be hemorrhages and/or microaneurysms $\geq$ standard photograph $2 \mathrm{~A}$ and/or soft exudates, venous beading, or intraretinal microvascular abnormalities definitely present and definition not met for severe nonproliferative retinopathy, early proliferative retinopathy, or highrisk proliferative retinopathy. Severe DR, including both proliferative and nonproliferative severe $\mathrm{DR}$, was defined as soft exudates, venous beading, and intraretinal microvascular abnormalities all definitely present in at least two of fields four through seven or two of the preceding three lesions present in at least two of fields four through seven and hemorrhages and microaneurysms present in these four fields, equaling or exceeding standard photo $2 \mathrm{~A}$ in at least one of them or intraretinal microvascular abnormalities present in each of fields four through seven equaling or exceeding standard photograph $8 \mathrm{~A}$ in at least two of them or presence of new vessels [31]. Any DR was defined as ETDRS severity score $\geq$ 14 in at least one eye and moderate DR was defined as ETDRS severity score $\geq 43$ in at least one eye. The definition of DR severity was based on the worse eye. Moderate and severe DR were combined into a single category (moderate) as the number of subjects with severe DR were too few for an adequately powered statistical analysis.

2.4. Assessment of Chronic Kidney Disease (CKD). Based on each individual marker, chronic kidney disease was defined as eGFR of $<60 \mathrm{~mL} /$ minute $/ 1.73 \mathrm{~m}^{2}$, using the US National Kidney Foundation Kidney Disease Outcome Quality Initiative Working Group definition [32]; GFR was estimated from the serum creatinine concentration $\left(\mathrm{eGFR}_{\mathrm{cr}}\right)$ [33] and serum cystatin $\mathrm{C}(\mathrm{eGFR}$ cys $)$ [34] using the recently developed CKD epidemiology collaboration (CKD-EPI) equations. We have earlier shown the prevalence of CKD by CKD-EPI equation to be similar to that of MDRD (modification of diet in renal disease) equation in all three major ethnic groups (Chinese, Malays, and Indians) in Singapore [35]. Creatinine concentrations were determined by the Jaffe method on the Beckman DxC800 analyzer with manufacturer provided calibrators traceable to SRM 967 [36]. Presence of albuminuria was defined as a urinary albumin : creatinine ratio of $30 \mathrm{mg} / \mathrm{g}$ $[9,37]$. Spot untimed urine samples were collected for measurement of albumin and creatinine. Albumin was measured in $\mathrm{mg} / \mathrm{L}$ and creatinine in $\mathrm{mmol} / \mathrm{L}$. The concentration ratio of urinary albumin to creatinine expressed in $\mu \mathrm{g} / \mathrm{mg}$ was used to estimate the total daily albumin excretion. Cystatin $\mathrm{C}$ was measured using particle-enhanced turbidimetric assay, and urine albumin was measured using a PEG-enhanced immunoturbidimetric method on the Siemens Advia platform at NUHS laboratory.

2.5. Statistical Analysis. Statistical analysis was performed with STATA version 13.0 (Texas, USA). A $P$ value of $<0.05$ was considered statistically significant. For univariate analysis, the Chi square test was performed for categorical variables and the independent $t$ test for continuous variables. The associations of CKD defined by each of the three markers alone and in combination $\left(\mathrm{eGFR}_{\mathrm{cr}}<60+\mathrm{eGFR}_{\mathrm{cys}}<60+\right.$ albuminuria) with moderate DR were examined using logistic regression models: first adjusted for age and gender, followed by adjustment for potential confounding factors including duration of diabetes, smoking, body mass index (BMI), systolic blood pressure, alcohol consumption, total cholesterol, and high-density lipoprotein cholesterol. Odds ratio (OR) and 95\% confidence interval (CI) were reported for the associations of CKD markers with moderate DR. Logistic regression models consisting of CKD markers in isolation and in combination were checked for goodness of fit with the Hosmer-Lemeshow test. To assess the performance of triple marker model including cystatin $\mathrm{C}$ in predicting moderate $\mathrm{DR}$, we constructed receiver operating characteristic (ROC) curves for each marker alone and in combination. The area under curve (AUC) for each model was compared with the null model without any CKD marker. In order to examine whether cystatin $\mathrm{C}$ is associated with $\mathrm{DR}$, independent of kidney function, we also examined the association between serum cystatin C levels (continuously and in quartiles) and moderate DR in separate logistic regression models. The models were similar to the main analysis, except for additional adjustment for $\mathrm{GFFR}_{\mathrm{cr}}$ and albuminuria in the multivariable models. Finally, we repeated the main analysis after excluding participants who reported having their diabetes diagnosed before the age of 35 years.

\section{Results}

A total of 1119 subjects with diabetes were enrolled into the study. Of these, $115(10.3 \%)$ had moderate DR in at least one eye. Table 1 shows the characteristics of subjects with and 
TABLE 1: Characteristics of participants by moderate DR status.

\begin{tabular}{|c|c|c|c|}
\hline & $\begin{array}{l}\text { No or mild DR } \\
\quad(n=1,004)\end{array}$ & $\begin{array}{l}\text { Moderate DR } \\
\quad(n=115)\end{array}$ & $P$ value ${ }^{*}$ \\
\hline Age (years) & $59.6(9.8)$ & $60.6(8.2)$ & 0.29 \\
\hline Women, \% & $454(45.2)$ & $49(42.6)$ & 0.59 \\
\hline Current smokers, $\%$ & $131(13.05)$ & $9(7.83)$ & 0.11 \\
\hline Alcohol drinkers, $\%$ & $140(13.9)$ & $10(8.7)$ & 0.12 \\
\hline Hypertension, \% & $686(68.5)$ & $92(80)$ & 0.01 \\
\hline Systolic blood pressure ( $\mathrm{mm} \mathrm{Hg}$ ) & $139(19)$ & $145(21)$ & 0.001 \\
\hline Diastolic blood pressure ( $\mathrm{mm} \mathrm{Hg}$ ) & $78(10)$ & $76(10)$ & 0.08 \\
\hline BMI $\left(\mathrm{kg} / \mathrm{m}^{2}\right)$ & $27.14(5.03)$ & $25.53(4.39)$ & 0.001 \\
\hline LDL-cholesterol & $3.07(0.95)$ & $3.02(0.10)$ & 0.63 \\
\hline HDL-cholesterol & $1.02(0.29)$ & $1.07(0.41)$ & 0.17 \\
\hline Blood glucose, $\mathrm{mmol} / \mathrm{L}$ & $9.51(4.22)$ & $12.08(6.29)$ & $<0.001$ \\
\hline $\mathrm{HbAlc}, \mathrm{mmol} / \mathrm{mol}$ & 59 & 69 & $<0.001$ \\
\hline HbAlc, (\%) & $7.5(1.5)$ & $8.5(2.0)$ & $<0.001$ \\
\hline Duration of diabetes, years & $9.5(8.2)$ & $17.0(10.5)$ & $<0.001$ \\
\hline $\mathrm{eGFR}_{\mathrm{cr}}\left(\mathrm{mL} / \mathrm{min} / 1.73 \mathrm{~m}^{2}\right)$ & $87(18)$ & $73(26)$ & $<0.001$ \\
\hline $\mathrm{eGFR}_{\text {cys }}\left(\mathrm{mL} / \mathrm{min} / 1.73 \mathrm{~m}^{2}\right)$ & $82(22)$ & $68(27)$ & $<0.001$ \\
\hline $\mathrm{ACR}(\mathrm{mg} / \mathrm{g})$ & $57.5(213)$ & $638.7(3055)$ & $<0.001$ \\
\hline
\end{tabular}

DR: diabetic retinopathy; BMI: body mass index; LDL: low density lipoprotein; HDL: high density lipoprotein; eGFR $_{\mathrm{cr}}$ : estimated glomerular filtration rate by serum creatinine; $\mathrm{eGFR}_{\text {cys }}$ : estimated glomerular filtration rate by serum cystatin C; ACR: albumin to creatinine ratio.

Data presented are proportions or means and standard deviation as appropriate for the variable.

${ }^{*} P$ value represents difference in characteristics by moderate DR status by analysis of variance or the chi-square test.

TABLE 2: Association between markers of CKD and moderate DR in the study population.

\begin{tabular}{|c|c|c|c|c|}
\hline & $\begin{array}{l}n \text { (cases) } \\
1119(115)\end{array}$ & Prevalence of moderate DR, \% & $\begin{array}{c}\text { Age, sex adjusted } \\
\text { OR }(95 \% \mathrm{CI})\end{array}$ & $\begin{array}{l}\text { Multivariable } \\
\text { OR }(95 \% \text { CI })^{*}\end{array}$ \\
\hline \multicolumn{5}{|l|}{$\mathrm{eGFR}_{\mathrm{cr}}$} \\
\hline$\geq 60$ & $991(81)$ & 8.2 & 1 (Reference) & 1 (Reference) \\
\hline$<60$ & $128(34)$ & 26.6 & $4.33(2.66-7.04)$ & $5.21(2.94-9.21)$ \\
\hline \multicolumn{5}{|l|}{$\mathrm{eGFR}_{\mathrm{cys}}$} \\
\hline$\geq 60$ & $894(68)$ & 7.6 & 1 (Reference) & 1 (Reference) \\
\hline$<60$ & $225(47)$ & 20.9 & $3.75(2.37-5.93)$ & $5.27(3.08-9.02)$ \\
\hline \multicolumn{5}{|l|}{ Albuminuria } \\
\hline No & $680(39)$ & 5.7 & 1 (Reference) & 1 (Reference) \\
\hline Yes & $324(76)$ & 23.4 & $4.05(2.69-6.10)$ & $2.50(1.58-3.96)$ \\
\hline \multicolumn{5}{|l|}{ CKD } \\
\hline None by all & $581(24)$ & 4.1 & 1 (Reference) & 1 (Reference) \\
\hline Any 1 marker & $379(48)$ & 12.7 & $3.59(2.15-6.01)$ & $2.33(1.33-4.11)$ \\
\hline Any 2 markers & $103(20)$ & 19.4 & $7.31(3.66-14.60)$ & $8.00(3.72-17.17)$ \\
\hline All 3 markers & $56(23)$ & 41.1 & $19.66(9.68-39.91)$ & $13.63(6.08-30.54)$ \\
\hline
\end{tabular}

Abbreviations: CI, confidence interval; OR, odds ratio; SD, standard deviation.

* Model adjusted for age (years), gender (men, women), body mass index $\left(\mathrm{kg} / \mathrm{m}^{2}\right)$, systolic blood pressure ( $\mathrm{mm} \mathrm{Hg}$ ), HbAlc (\%) and diabetes duration (years).

without moderate DR. Persons with moderate DR had higher prevalence of hypertension, had higher levels of systolic BP, BMI, blood glucose, HbAlc, and ACR and longer duration of diabetes and had lower levels of eGFR (both $\mathrm{eGFR}_{\mathrm{cr}}$ and $\mathrm{GGF}_{\text {cys }}$ ). The prevalence of CKD defined by creatinine, cystatin C, and albuminuria in our cohort was $11.4 \%, 20.1 \%$, and $29.0 \%$, respectively. In subjects with CKD defined by $\mathrm{eGFR}_{\mathrm{cr}}<60 \mathrm{~mL} / \mathrm{min} / 1.73 \mathrm{~m}^{2}, 86.7 \%(n=111)$ also had
$\mathrm{eGFR}_{\text {cys }}<60 \mathrm{~mL} / \mathrm{min} / 1.73 \mathrm{~m}^{2}$ and $46.0 \%(n=59)$ had ACR $\geq 30 \mathrm{mg} / \mathrm{g}$. In subjects without CKD defined by eGFR ${ }_{\mathrm{cr}}(n=$ 991), $11.5 \%(n=114)$ had $\mathrm{eGFR}_{\text {cys }}<60 \mathrm{~mL} / \mathrm{min} / 1.73 \mathrm{~m}^{2}$ and $34.4 \%(n=341)$ had ACR $\geq 30 \mathrm{mg} / \mathrm{g}$. Five percent $(n=56)$ of adults had CKD defined by all 3 markers.

Table 2 shows the results of multivariate analysis performed to assess the association between each marker in isolation and in combination. The prevalence of moderate DR 
TABLE 3: Area under curve (AUC) for markers of CKD in isolation and in combination for discriminating persons with/without moderate DR.

\begin{tabular}{|c|c|c|c|}
\hline & AUC & $95 \% \mathrm{CI}$ & $P^{*}$ \\
\hline Null model & 0.790 & $0.748-0.832$ & - \\
\hline $\mathrm{eGFR}_{\mathrm{cr}}$ only & 0.813 & $0.775-0.852$ & 0.09 \\
\hline $\mathrm{eGFR}_{\text {cys }}$ only & 0.816 & $0.776-0.857$ & 0.07 \\
\hline Albuminuria only & 0.808 & $0.768-0.849$ & 0.07 \\
\hline $\mathrm{eGFR}_{\mathrm{cr}}+\mathrm{eGFR}_{\mathrm{cys}}$ & 0.821 & $0.781-0.860$ & 0.05 \\
\hline $\mathrm{eGFR}_{\mathrm{cr}}+$ albuminuria & 0.828 & $0.791-0.864$ & 0.01 \\
\hline $\mathrm{eGFR}_{\mathrm{cys}}+$ albuminuria & 0.828 & $0.789-0.868$ & 0.015 \\
\hline $\mathrm{eGFR}_{\mathrm{cr}}+\mathrm{eGFR}_{\mathrm{cys}}+$ albuminuria & 0.834 & $0.795-0.871$ & 0.0089 \\
\hline
\end{tabular}

CI: confidence interval.

Null model: age, gender, body mass index, systolic BP, HbAlc, and diabetes duration.

${ }^{*} P$ value, compared with null model.

was higher among those with CKD-eGFR $\mathrm{cr}_{\mathrm{c}}(26.6 \%)$ followed by albuminuria (23.4\%) and CKD-eGFR cys $(20.9 \%)$. In separate models, $\mathrm{CKD}-\mathrm{eGFR}$ r, CKD-eGFR $_{\mathrm{cys}}$, and albuminuria were significantly associated with DR with ORs ranging from 2.50 for albuminuria to 5.21 for CKD-eGFR ${ }_{c r}$. Subjects with CKD defined by triple markers were fourteen times more likely to have moderate DR (OR (95\% CI) $=13.63(6.08-$ 30.54)) compared to those without CKD defined by any marker.

Table 3 shows the AUC for diagnostic models consisting of CKD markers in isolation and in combination, compared to the null model without CKD markers. Diagnostic models containing CKD defined by any of the 3 markers (creatinine, cystatin $\mathrm{C}$, and ACR) alone were not significantly better than the null model $(P=0.09,0.07$, and 0.07 , resp.). In the models combining 2 markers, CKD defined by a combination of creatinine and albuminuria (AUC $0.828, P=0.01$ ) or a combination of cystatin C and albuminuria (AUC 0.828, $P=0.02$ ) had better discrimination than the null model. The model using triple markers had the highest AUC of 0.834 and was strongly significant for better discrimination than the null model $(P=0.0089$, Figure 2$)$. When the triple marker model was compared to single and double marker models, incremental diagnostic value was significantly better than the cystatin $\mathrm{C}$ model $(P=0.035)$ but not for the other models. The association of serum cystatin $C$ levels with moderate DR is shown in Table 4. The prevalence of moderate DR increased with increasing quartiles of cystatin C with nearly half of the participants in the highest quartile having moderate DR (48.7\%). Cystatin C was significantly associated with moderate DR in both quartile and continuous analysis independent of $\mathrm{eGFR}_{\mathrm{cr}}$ and ACR. Finally, repeating the main analysis in Table 2, after excluding those with diabetes diagnosed before the age of 35 years $(n=53$ excluded), did not alter the effect estimates. For example, compared to those without CKD by any marker, the OR $(95 \%$ CI) of moderate DR was 2.23 (1.19-4.20) by any one marker; 6.99 (3.14-15.58) by any 2 markers; and $13.04(5.67-30.00)$ by any 3 markers (data not shown in the table).

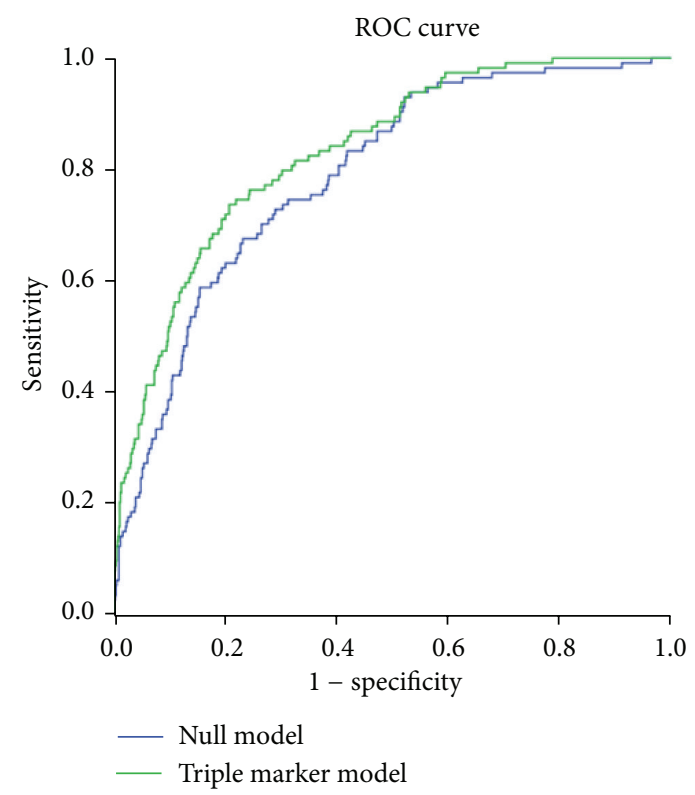

FIGURE 2: Receiver operating characteristic (ROC) curves comparing the null model (age, gender, body mass index, systolic BP, HbAlc, and diabetes duration) with the triple marker model $\left(\mathrm{eGFR}_{\mathrm{cr}}+\right.$ $\mathrm{eGFR}_{\text {cys }}+$ albuminuria, in addition to the variables included in the null model).

\section{Discussion}

In a population-based sample of Indian adults with diabetes, CKD defined by all 3 markers including eGFR $r$, eGFR cys $_{\text {, }}$, and albuminuria was strongly associated with moderate DR independent of other cardiovascular risk factors. In addition, elevated levels of cystatin C $(\geq 1.12 \mathrm{mg} / \mathrm{L})$ were associated with more than 3-fold odds of moderate DR independent of albuminuria and $\mathrm{eGFR}_{\mathrm{cr}}$.

The prevalence of CKD in the current study including persons with diabetes was $11.4 \%$ by eGFR cr and $20.1 \%$ by $\mathrm{eGFR}_{\text {cys }} .5 \%$ had CKD by the triple marker panel. This is similar to previous reports from the US. In the NHANES cohort, the prevalence of reduced kidney function was $16.5 \%$ using eGFR cr and $22 \%$ using $\mathrm{eGFR}_{\text {cys }}$ among persons with diabetes [38]. In the REGARDS cohort consisting of subjects with and without diabetes, $12.9 \%$ had reduced kidney function using eGFR $\mathrm{G}_{\text {cys }}, 10.9 \%$ had reduced kidney function using $\mathrm{eGFR}_{\mathrm{cr}}$, and $14.8 \%$ had albuminuria. Only $3.3 \%$ had CKD defined by all 3 markers [23].

Several studies have shown associations between CKD defined by a single marker including creatinine [10, 3941] or albuminuria $[10,41]$ and DR. Few previous studies have shown that CKD defined by double markers including $\mathrm{eGFR}_{\mathrm{cr}}$ and albuminuria is strongly associated with DR compared to eGFR $\mathrm{cr}_{\mathrm{c}}$ alone. Chen et al. found CKD defined by eGFR ${ }_{c r}$ to be associated with DR only in the presence of albuminuria [6]. Similarly, in a recent cross-sectional study involving a multiethnic Asian population, we found that CKD was associated with DR only in the presence of albuminuria [9]. Penno et al. found albuminuric CKD phenotypes with reduced eGFR $_{\mathrm{cr}}$ to be strongly associated with advanced 
TABLE 4: Association between serum cystatin C and moderate DR.

\begin{tabular}{lccc}
\hline Cystatin C & Prevalence of DR & $\begin{array}{c}\text { Age-gender adjusted OR } \\
(95 \% \text { CI })\end{array}$ & $\begin{array}{c}{ }^{*} \text { Multivariable adjusted OR } \\
(95 \% \text { CI })\end{array}$ \\
\hline Q1 $(<0.82)$ & $19(16.5 \%)$ & 1 (Reference) & $1($ Reference $)$ \\
Q2 $(0.82-0.94)$ & $21(18.3 \%)$ & $1.08(0.57-2.07)$ & $1.05(0.51-2.16)$ \\
Q3 $(0.95-1.11)$ & $19(16.5 \%)$ & $0.99(0.50-1.94)$ & $1.00(0.46-2.17)$ \\
Q4 $(1.12-5.07)$ & $56(48.7 \%)$ & $3.51(1.94-6.38)$ & $3.38(1.55-7.38)$ \\
Per SD increase & - & $1.92(1.59-2.33)$ & $1.90(1.37-2.61)$ \\
\hline
\end{tabular}

DR: diabetic retinopathy; Q: quartiles; SD: standard deviation.

${ }^{*}$ Model adjusted for age (years), gender (men, women), body mass index $\left(\mathrm{kg} / \mathrm{m}^{2}\right)$, systolic blood pressure (mm Hg), HbAlc (\%), diabetes duration (years), albumin creatinine ratio $(\mathrm{mg} / \mathrm{g})$, and $\mathrm{eGFR}_{\mathrm{cr}}\left(\mathrm{mL} / \mathrm{min} / 1.73 \mathrm{~m}^{2}\right)$.

DR (OR 2.97), compared to both albuminuric CKD without reduced $\mathrm{eGFR}_{\mathrm{cr}}$ and nonalbuminuric CKD phenotypes [10].

Recently, CKD outcome studies have established that cystatin $\mathrm{C}$ and albuminuria can improve risk stratification among persons with CKD defined by $\mathrm{eGFR}_{\mathrm{cr}}$, with respect to mortality risk, cardiovascular disease, heart failure, and end stage renal disease [22, 42-44]. In the reasons for geographic and racial differences in stroke (REGARDS) cohort, allcause mortality was higher in persons with CKD defined by $\mathrm{eGFR}_{\mathrm{cr}}$ who also had albuminuria [43] or CKD based on eGFR cys [22]. More recently, Peralta et al. evaluated a triple marker approach for the detection and classification of CKD using creatinine, cystatin C, and ACR [23]. They found the highest risk for all-cause mortality and incident end-stage renal disease among those with CKD defined by all markers compared to those with CKD defined by creatinine alone. Their findings confirmed that a triple marker approach for risk classification in persons with CKD increased the predictive accuracy for all-cause mortality and end-stage renal disease compared to the traditional creatinine based definition of CKD alone. In the current study, for the first time, we have shown a triple marker panel of defining CKD to have a stronger association with DR compared to single or double marker panels. Whether the triple marker approach also increases the predictive value for incident DR or progression of DR remains to be determined in future longitudinal studies.

Compared to CKD defined by serum creatinine, CKD defined by serum cystatin $C$ appears to better predict and risk stratify patients with diabetes for end stage renal disease [20, 21]. In the National Health and Nutrition Examination Survey (NHANES), Tsai et al. found a higher prevalence of reduced kidney function among persons with diabetes using eGFR compared to $\mathrm{GFFR}_{\mathrm{cr}}$ [38]. In the same study, $\mathrm{eGFR}_{\text {cys }}$ was strongly associated with diabetic complications, including DR [38]. It is possible that cystatin C could also be a better predictor for DR compared to $\mathrm{eGFR}_{\mathrm{cr}}$ and albuminuria, related to shared pathogenic pathways between retinopathy and cystatin C [45]. In He et al's study, the prevalence of sight threatening DR (STDR) was highest in the 4th quartile $(\sim 60 \%, P<0.01)$ and cystatin $C$ was found to be an independent risk factor for STDR together with HbAlc and diabetes duration. An 11-fold risk of STDR was conferred when serum cystatin C levels were more than $1.25 \mathrm{mg} / \mathrm{L}$ [27]. In our study, higher levels of serum cystatin $\mathrm{C}$ showed a significant association with DR independent of eGFR $\mathrm{Cr}_{\mathrm{cr}}$ and albuminuria. This suggests that cystatin $\mathrm{C}$ may also play a direct role in DR, independent of kidney function, by promoting vascular endothelial growth factor (VEGF) driven angiogenesis [45]. Further studies are required to determine the concordance of cystatin C defined CKD phenotypes with DR.

The relatively small number of subjects with CKD defined by triple markers is a limitation of our study, resulting in an imprecise confidence interval estimate $(\mathrm{OR}(95 \% \mathrm{CI})=13.63$ (6.08-30.54)). However, despite the smaller sample size, our effect estimates for CKD by triple marker remained highly statistically significant due to a large effect size. Increasing the sample size can only strengthen our conclusions. The crosssectional nature of our study is another limitation of our study. Although a prospective study would be ideal to assess the risk of DR progression, the use of triple markers to predict the presence of moderate to severe DR may still prove to be useful to patients and clinicians without easy access to fundus photography or an ophthalmologist.

In conclusion, CKD defined by a triple marker panel was strongly associated with moderate DR in Indian adults with diabetes. If confirmed by future prospective studies and in other populations, a triple marker approach may have implications on changing clinical practice to incorporate cystatin $\mathrm{C}$ to improve risk stratification of DR in persons with CKD. This group of high-risk individuals may benefit from closer surveillance and more timely intervention before the onset of irreversible sight threatening complications.

\section{Conflict of Interests}

The authors declare that there is no conflict of interests regarding the publication of this paper.

\section{Acknowledgment}

This study was supported by the Ministry of Education, Academic Research Fund Tier 1 No. T1, February 01, 2012, National Medical Research Council Grants STaR/0003/2008 and NMRC/TA/0008/2012.

\section{References}

[1] J. W. Y. Yau, S. L. Rogers, R. Kawasaki et al., "Global prevalence and major risk factors of diabetic retinopathy," Diabetes Care, vol. 35, no. 3, pp. 556-564, 2012. 
[2] D. Jee, W. K. Lee, and S. Kang, "Prevalence and risk factors for diabetic retinopathy: the Korea National Health and Nutrition Examination Survey 2008-2011," Investigative Ophthalmology and Visual Science, vol. 54, no. 10, pp. 6827-6833, 2013.

[3] F. H. Wang, Y. B. Liang, F. Zhang et al., "Prevalence of diabetic retinopathy in rural China: the Handan Eye Study," Ophthalmology, vol. 116, no. 3, pp. 461-467, 2009.

[4] Y. Zheng, E. L. Lamoureux, R. Lavanya et al., "Prevalence and risk factors of diabetic retinopathy in migrant Indians in an urbanized society in Asia: the Singapore Indian eye study," Ophthalmology, vol. 119, no. 10, pp. 2119-2124, 2012.

[5] L. Chen, D. J. Magliano, and P. Z. Zimmet, "The worldwide epidemiology of type 2 diabetes mellitus-present and future perspectives," Nature Reviews Endocrinology, vol. 8, no. 4, pp. 228-236, 2012.

[6] Y.-H. Chen, H.-S. Chen, and D.-C. Tarng, "More impact of microalbuminuria on retinopathy than moderately reduced GFR among type 2 diabetic patients," Diabetes Care, vol. 35, no. 4, pp. 803-808, 2012.

[7] K. J. Cruickshanks, L. L. Ritter, R. Klein, and S. E. Moss, "The association of microalbuminuria with diabetic retinopathy: the Wisconsin Epidemiologic Study of Diabetic Retinopathy," Ophthalmology, vol. 100, no. 6, pp. 862-867, 1993.

[8] P. K. Rani, R. Raman, A. Gupta, S. S. Pal, V. Kulothungan, and T. Sharma, "Albuminuria and diabetic retinopathy in type 2 diabetes mellitus sankara nethralaya diabetic retinopathy epidemiology and molecular genetic study (SN-DREAMS, report 12)," Diabetology \& Metabolic Syndrome, vol. 3, no. 1, article 9, 2011.

[9] C. Sabanayagam, V. H. X. Foo, M. K. Ikram et al., "Is chronic kidney disease associated with diabetic retinopathy in Asian adults?" Journal of Diabetes, vol. 6, pp. 556-563, 2014.

[10] G. Penno, A. Solini, G. Zoppini et al., "Rate and determinants of association, between advanced retinopathy and chronic kidney disease in patients with type 2 diabetes: the renal insufficiency and cardiovascular events (RIACE) Italian multicenter study," Diabetes Care, vol. 35, no. 11, pp. 2317-2323, 2012.

[11] C. W. Wong, T. Y. Wong, C.-Y. Cheng, and C. Sabanayagam, "Kidney and eye diseases: common risk factors, etiological mechanisms, and pathways," Kidney International, vol. 85, pp. 1290-1302, 2014.

[12] H. Cho and L. Sobrin, "Genetics of diabetic retinopathy," Current Diabetes Reports, vol. 14, no. 8, article 515, 2014.

[13] J. Z. Kuo, T. Y. Wong, J. I. Rotter, and J. L. Wiggs, "Challenges in elucidating the genetics of diabetic retinopathy," JAMA Ophthalmology, vol. 132, no. 1, pp. 96-107, 2014.

[14] M. G. Pezzolesi, G. D. Poznik, J. C. Mychaleckyj et al., “Genomewide association scan for diabetic nephropathy susceptibility genes in type 1 diabetes," Diabetes, vol. 58, no. 6, pp. 1403-1410, 2009.

[15] S. Maeda, S.-I. Araki, T. Babazono et al., "Replication study for the association between four loci identified by a genome-wide association study on European American subjects with type 1 diabetes and susceptibility to diabetic nephropathy in Japanese subjects with type 2 diabetes," Diabetes, vol. 59, no. 8, pp. 20752079, 2010.

[16] M. Chen, W.-R. Lin, C.-H. Lu et al., "Chimerin 2 genetic polymorphisms are associated with non-proliferative diabetic retinopathy in Taiwanese type 2 diabetic patients," Journal of Diabetes and Its Complications, vol. 28, pp. 460-463, 2014.
[17] C. Hu, R. Zhang, W. Yu et al., " $C P V L / C H N 2$ genetic variant is associated with diabetic retinopathy in Chinese type 2 diabetic patients," Diabetes, vol. 60, no. 11, pp. 3085-3089, 2011.

[18] M. Buraczynska, P. Wacinski, P. Zukowski, M. Dragan, and A. Ksiazek, "Common polymorphism in the cannabinoid type 1 receptor gene (CNR1) is associated with microvascular complications in type 2 diabetes," Journal of Diabetes and Its Complications, vol. 28, no. 1, pp. 35-39, 2014.

[19] A. Akhter, K. Fatema, S. F. Ahmed, A. Afroz, L. Ali, and A. Hussain, "Prevalence and associated risk indicators of retinopathy in a rural Bangladeshi population with and without diabetes," Ophthalmic Epidemiology, vol. 20, no. 4, pp. 220-227, 2013.

[20] A. S. Krolewski, J. H. Warram, C. Forsblom et al., "Serum concentration of cystatin $\mathrm{C}$ and risk of end-stage renal disease in diabetes," Diabetes Care, vol. 35, no. 11, pp. 2311-2316, 2012.

[21] M. E. Pavkov, W. C. Knowler, R. L. Hanson et al., "Comparison of serum cystatin $\mathrm{C}$, serum creatinine, measured GFR, and estimated GFR to assess the risk of kidney failure in American Indians with diabetic nephropathy," The American Journal of Kidney Diseases, vol. 62, no. 1, pp. 33-41, 2013.

[22] C. A. Peralta, R. Katz, M. J. Sarnak et al., "Cystatin C identifies chronic kidney disease patients at higher risk for complications," Journal of the American Society of Nephrology, vol. 22, no. 1, pp. 147-155, 2011.

[23] C. A. Peralta, M. G. Shlipak, S. Judd et al., "Detection of chronic kidney disease with creatinine, cystatin c, and urine albuminto-creatinine ratio and association with progression to endstage renal disease and mortality," Journal of the American Medical Association, vol. 305, no. 15, pp. 1545-1552, 2011.

[24] M. G. Shlipak, M. J. Sarnak, R. Katz et al., "Cystatin C and the risk of death and cardiovascular events among elderly persons," The New England Journal of Medicine, vol. 352, no. 20, pp. 20492060, 2005.

[25] L. A. Stevens, J. Coresh, C. H. Schmid et al., "Estimating GFR using serum cystatin $\mathrm{C}$ alone and in combination with serum creatinine: a pooled analysis of 3,418 individuals with CKD," American Journal of Kidney Diseases, vol. 51, no. 3, pp. 395-406, 2008.

[26] M. G. Shlipak, R. Katz, M. J. Sarnak et al., "Cystatin C and prognosis for cardiovascular and kidney outcomes in elderly persons without chronic kidney disease," Annals of Internal Medicine, vol. 145, no. 4, pp. 237-246, 2006.

[27] R. He, J. Shen, J. Zhao et al., "High cystatin C levels predict severe retinopathy in type 2 diabetes patients," European Journal of Epidemiology, vol. 28, no. 9, pp. 775-778, 2013.

[28] R. Lavanya, V. S. E. Jeganathan, Y. Zheng et al., "Methodology of the Singapore Indian Chinese Cohort (SICC) eye study: quantifying ethnic variations in the epidemiology of eye diseases in Asians," Ophthalmic Epidemiology, vol. 16, no. 6, pp. 325-336, 2009.

[29] American Diabetes Association, "Standards of medical care in diabetes-2009," Diabetes Care, vol. 32, supplement 1, pp. S13S61, 2008.

[30] D. Rooney, W. K. Lye, G. Tan et al., "Body mass index and retinopathy in Asian populations with diabetes mellitus," Acta Diabetologica, 2014.

[31] "Grading diabetic retinopathy from stereoscopic color fundus photographs - an extension of the modified Airlie House classification. ETDRS report number 10. Early Treatment Diabetic Retinopathy Study Research Group," Ophthalmology, vol. 98, no. 5, pp. 786-806, 1991. 
[32] National Kidney Foundation, "K/DOQI clinical practice guidelines for chronic kidney disease: evaluation, classification, and stratification," American Journal of Kidney Diseases, vol. 39, no. 2, supplement 1, pp. S1-S266, 2002.

[33] A. S. Levey, L. A. Stevens, C. H. Schmid et al., "A new equation to estimate glomerular filtration rate," Annals of Internal Medicine, vol. 150, no. 9, pp. 604-612, 2009.

[34] L. A. Inker, C. H. Schmid, H. Tighiouart et al., "Estimating glomerular filtration rate from serum creatinine and cystatin C," The New England Journal of Medicine, vol. 367, no. 1, pp. 20-29, 2012.

[35] C. Sabanayagam, T. Y. Wong, and E. S. Tai, “The CKD-EPI equation and MDRD study equation find similar prevalence of chronic kidney disease in asian populations," Annals of Internal Medicine, vol. 151, no. 12, pp. 892-893, 2009.

[36] B. W. Teo, C. Sabanayagam, J. Liao et al., "Comparison of CKD-EPI cystatin $\mathrm{C}$ and creatinine glomerular filtration rate estimation equations in asian indians," International Journal of Nephrology, vol. 2014, Article ID 746497, 8 pages, 2014.

[37] KDOQI, "KDOQI clinical practice guidelines and clinical practice recommendations for diabetes and chronic kidney disease," American Journal of Kidney Diseases, vol. 49, no. 2, supplement 2, pp. S12-S154, 2007.

[38] C.-W. Tsai, M. E. Grams, L. A. Inker, J. Coresh, and E. Selvin, "Cystatin C- and creatinine-based estimated glomerular filtration rate, vascular disease, and mortality in persons with diabetes in the U.S," Diabetes Care, vol. 37, no. 4, pp. 1002-1008, 2014.

[39] J. E. Grunwald, J. Alexander, M. Maguire et al., "Prevalence of ocular fundus pathology in patients with chronic kidney disease," Clinical Journal of the American Society of Nephrology, vol. 5, no. 5, pp. 867-873, 2010.

[40] G. Liew, P. Mitchell, T. Y. Wong, and J. J. Wang, "Retinal microvascular signs are associated with chronic kidney disease in persons with and without diabetes," Kidney \& Blood Pressure Research, vol. 35, no. 6, pp. 589-594, 2012.

[41] C. Sabanayagam, A. Shankar, D. Koh et al., "Retinal microvascular caliber and chronic kidney disease in an Asian population," American Journal of Epidemiology, vol. 169, no. 5, pp. 625-632, 2009.

[42] M. Tonelli, P. Muntner, A. Lloyd et al., "Using proteinuria and estimated glomerular filtration rate to classify risk in patients with chronic kidney disease: a cohort study," Annals of Internal Medicine, vol. 154, no. 1, pp. 12-21, 2011.

[43] D. G. Warnock, P. Muntner, P. A. McCullough et al., "Kidney function, albuminuria, and all-cause mortality in the REGARDS (Reasons for Geographic and Racial Differences in Stroke) study," American Journal of Kidney Diseases, vol. 56, no. 5, pp. 861-871, 2010.

[44] Chronic Kidney Disease Prognosis Consortium, K. Matsushita, M. van der Velde et al., "Association of estimated glomerular filtration rate and albuminuria with all-cause and cardiovascular mortality in general population cohorts: a collaborative metaanalysis," The Lancet, vol. 375, pp. 2073-2081, 2010.

[45] E. Im, A. Venkatakrishnan, and A. Kazlauskas, "Cathepsin B regulates the intrinsic angiogenic threshold of endothelial cells," Molecular Biology of the Cell, vol. 16, no. 8, pp. 3488-3500, 2005. 


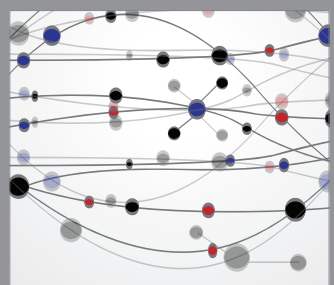

The Scientific World Journal
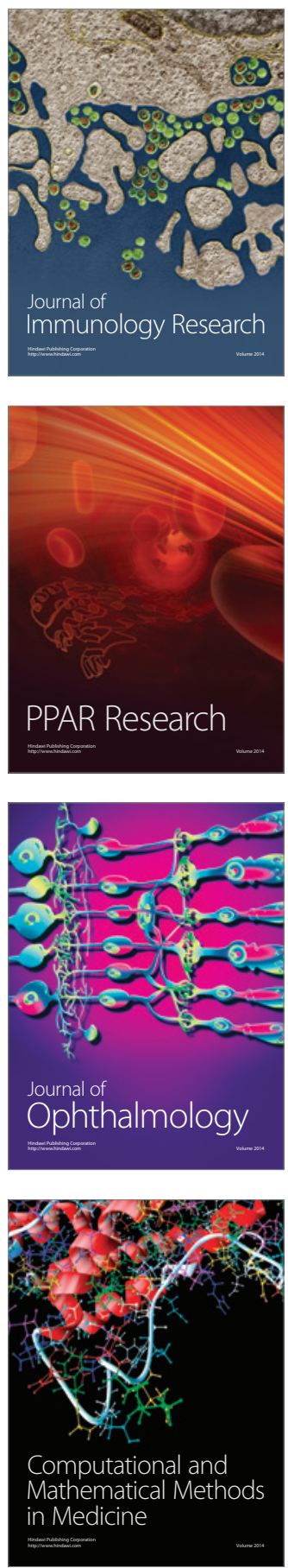

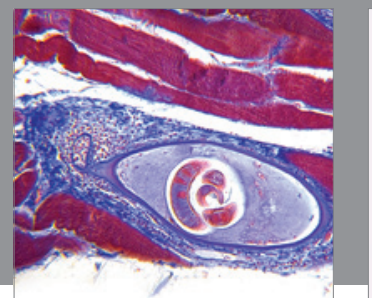

Gastroenterology

Research and Practice
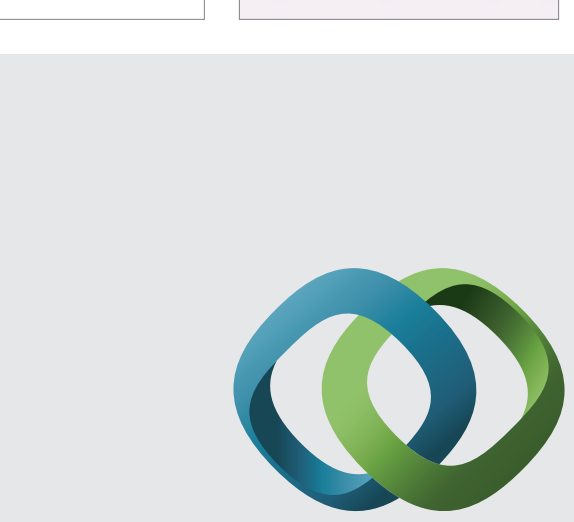

\section{Hindawi}

Submit your manuscripts at

http://www.hindawi.com
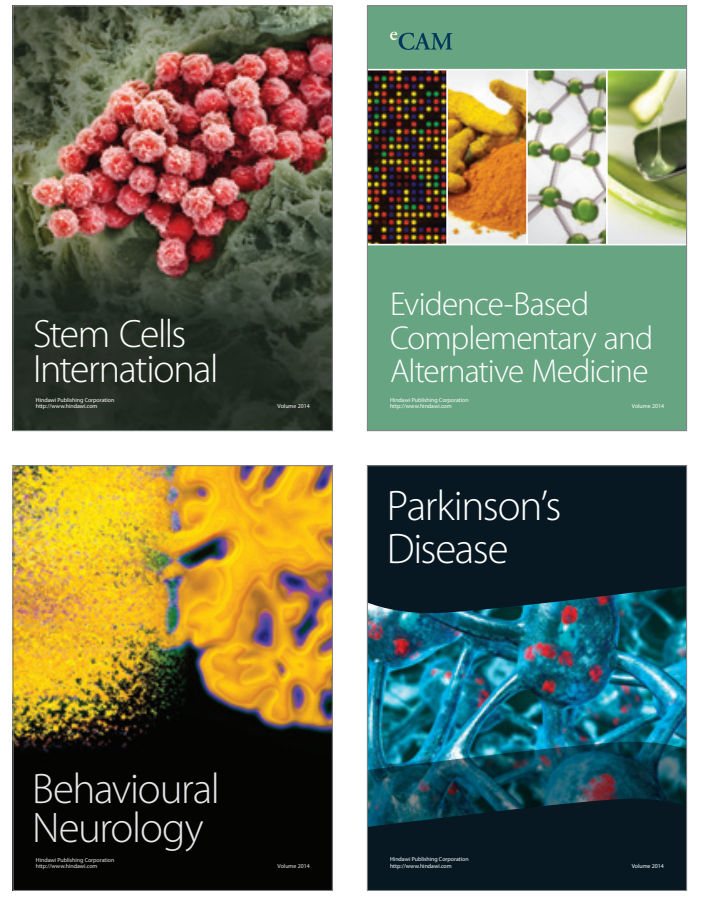
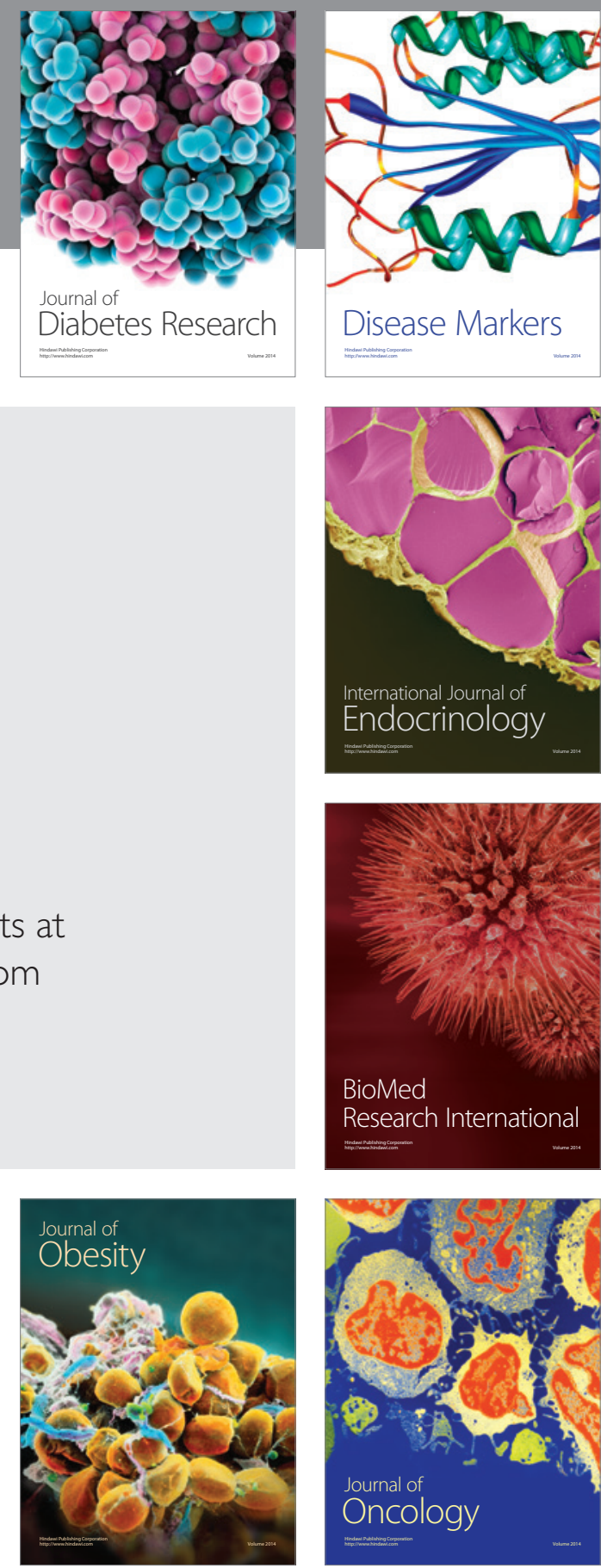

Disease Markers
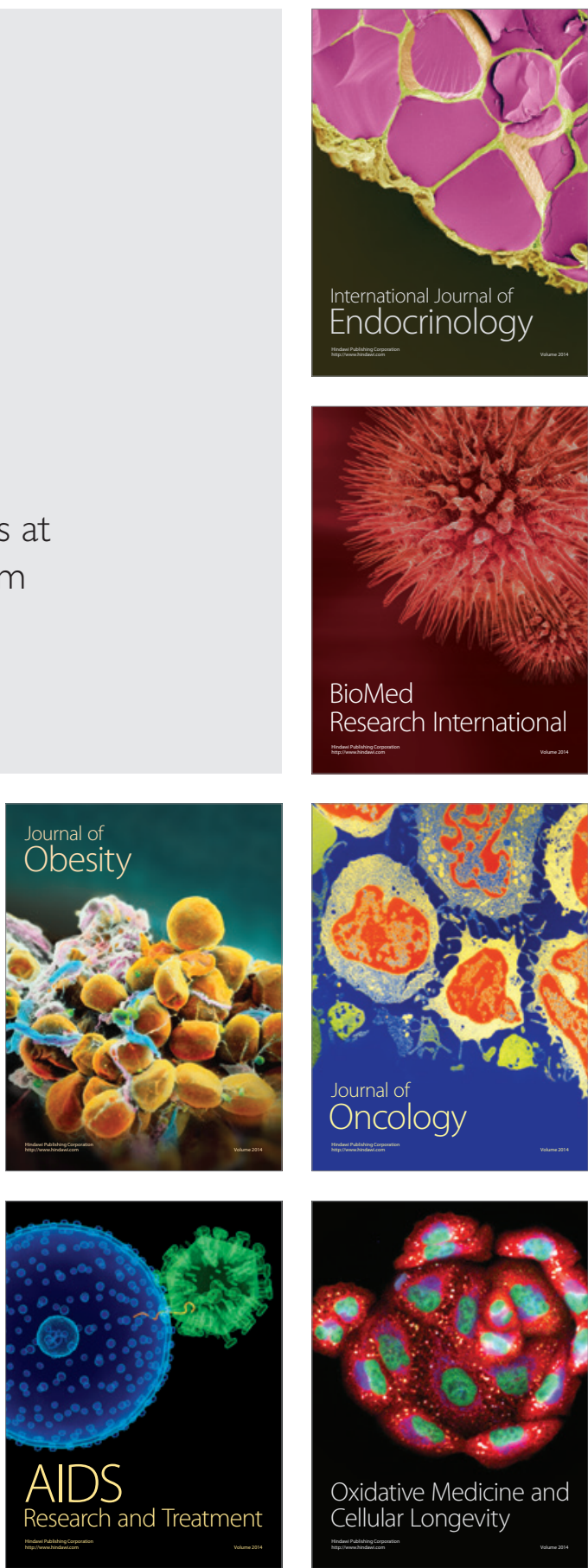